\title{
Qualität in der Onkologie
}

\section{Jürg Nadig}

Dr. med., Präsident Schweizerische Gesellschaft für Medizinische Onkologie (SGMO)

\author{
Der Entwurf eines Nationalen Krebsregistergesetzes (KRG) liegt dem Parlament zur \\ Beratung vor. Auch wenn Register nicht alle Fragen beantworten, verdienen sie die \\ Unterstützung der Ärzteschaft, schaffen sie doch die gesetzlichen Grundlagen, um \\ Fragen zur Versorgungsqualität beantworten zu können.
}

Der Entwurf eines Nationalen Krebsregistergesetzes (KRG) liegt dem Parlament zur Beratung vor. Sicher keine einfache Materie, gilt es doch, verschiedene Aufgaben in einem Gesetz zu vereinen. Sektorübergreifende Behandlungspfade und der Entwurf zu einem Nationalen KRG sind aber wesentliche Elemente, um über Qualität und Kosten das Gesundheitswesen zu steuern [1, 2]. Deshalb braucht es im KRG neben epidemiologischen Daten auch Behandlungsdaten, die den Fachgesellschaften für Qualitätsregister zur Verfügung stehen [3]. Basierend auf diesen Daten liesse sich beispielsweise prüfen, ob auch in der Schweiz, wie in den USA, die Behandlung in forschenden Institutionen besser ist. Oder aber, ob bei uns diese Differenz fehlt, da genügend finanzielle Mittel für eine qualitativ hochwertige Behandlung zur Verfügung stehen.
Korrespondenz: Dr. med. Jürg Nadig, MAE Bannhaldenstrasse 7 CH-8180 Bülach juerg.nadig[at]hin.ch

\section{Vollständige Informationen notwendig}

Während der Behandlungspfad aufzeigt, was heute zum Minimalstandard gehört, können Registerdaten zeigen,

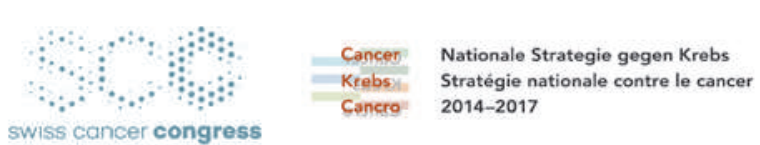

\section{Schweizer Krebskongress}

Am 27. August 2015 findet an der Universität Freiburg der 2. Schweizer Krebskongress statt. Der vor einem Jahr von den Leistungserbringern in der Krebsbehandlung initiierte Kongress wird in diesem Jahr zusammen mit Akteuren der Nationalen Strategie gegen Krebs durchgeführt.

Der Schweizer Krebskongress versteht sich als Think Tank, jährliches Arbeitstreffen und Forum zur kontinuierlichen Verbesserung von Vorsorge, Behandlung, Betreuung und Forschung in der Onkologie. Die Veranstaltung soll die multidisziplinäre und interprofessionelle Vernetzung der verschiedenen Leistungserbringer und Organisationen fördern. Hauptthema des diesjährigen Kongresses ist die Qualität in der Onkologie. Im Zentrum der Tagung stehen parallel geführte thematische Workshops, in denen die Teilnehmenden bestimmte Qualitätsaspekte vertiefen und diskutieren können. Zudem ist ein Update zur Nationalen Strategie gegen Krebs geplant.

Eingeladen sind Ärzte, Pflegende und alle Fachpersonen, die an der Behandlung und Betreuung von Krebsbetroffenen beteiligt sind. Weitere Informationen / Online-Registrierung auf www.swisscancercongress.ch wie viele Betroffene eine evidenzbasierte (Guidelinegemässe) Therapie erhalten und inwiefern dadurch ihr Überleben ausserhalb klinischer Studien beeinflusst wird [4]. Dafür brauchen die kantonalen Krebsregister aber detaillierte Informationen zur Diagnose und effektiv durchgeführten Behandlung. Eine nutzerfreundliche Datenerfassung und klar definierte Datenflüsse sind zentral, um die Daten sorgfältig aufbereiten zu können. Da der Krankheitsverlauf durch die zeitlich gestaffelten Therapien mit beeinflusst wird, müssen die Daten über die ganze Behandlungsdauer erfasst und lange genug aufbewahrt werden.

\section{Viele Fragen noch offen}

Doch wie lässt sich bei der zunehmenden Zahl von Patienten mit Mehrfacherkrankungen eine «Guidelinegemässe Behandlung» überhaupt umsetzen, wenn zudem der Patient zur gemeinsamen Entscheidungsfindung (Shared Decision Making) eingeladen werden soll? Sind Register überhaupt in der Lage, solche Fragen zu beantworten? Wäre dann eine Zielerreichung oder eine Guideline-gemässe Behandlung höher zu werten? Auch wenn Register nicht alle Fragen beantworten können, verdient der vorliegende Gesetzesentwurf die Unterstützung der Ärzteschaft, weil er in einem Teilbereich der Tumorerkrankung die gesetzlichen Grundlagen dazu schafft, basierend auf einer soliden Datengrundlage auch Qualitätsprojekte der Fachgesellschaften zu initiieren und Fragen zur Versorgungsqualität zu beantworten.

1 Bosshard C, Meyer-Nikolic VA, Nadig J, Pfisterer J. Pilotprojekt «Sektorübergreifender Behandlungspfad Kolonkarzinom». Schweiz Ärztezeitung. 2014;95(31-32):1136-7.

2 www.bag.admin.ch/themen/gesundheitspolitik/10374/index. html?lang=de\&download=NHzLpZeg7t,lnp6IONTU042l2Z6ln1acy 4Zn4Z2qZpnO2Yuq2Z6gpJCMdYR_gWym162epYbg2c_ JjKbNoKSn6A--

3 Nadig J, Gruber G. Qualitätsregister der Fachgesellschaften als Beitrag zum NKP II. Schweizer Krebsbulletin 32. 71; 2013.

4 Nadig J, Gähler E. Guidelines taugen nicht zu Wirtschaftlichkeitsverfahren. Schweiz Ärztezeitung. 2011;92(43):1660-2. 